\title{
Paget Disease of the Vulva
}

National Cancer Institute

\section{Source}

National Cancer Institute. Paget Disease of the Vulva. NCI Thesaurus. Code C4027.

An uncommon intraepithelial malignant neoplasm of eccrine or apocrine origin, arising from the vulva. It usually affects post-menopausal women. In approximately $10-20 \%$ of the cases there is an associated anorectal, or urothelial carcinoma or a skin appendage adenocarcinoma identified. It presents as a red, eczematous lesion. Microscopically, it is characterized by the presence of the typical Paget cells which are large, round cells with abundant cytoplasm and prominent nuclei. 